\title{
New treatment for sarcoidosis: where's the proof?
}

\author{
R.P. Baughman, E.E. Lower
}

This is an exciting time for clinicians treating sarcoidosis, since many agents are being proposed for therapy. These range from the old standby corticosteroids to immunomodulators such as methotrexate, hydroxychloroquine, chlorambucil, cyclophosphamide, cyclosporine, thalidomide, and azathioprine [1]. The problem: none of these drugs is perfect for all patients. Although this dilemma is obvious to the patient and to the physician, it is not always clear to the researcher.

The initial reports of a new therapy usually discuss its effectiveness for patients who have not been successfully treated by other drugs, or for those who have refused to take corticosteroids. These reports of single cases should be tempered by the variable course of sarcoidosis. At initial presentation, some of the "sickest" patients will present with erythema nodosum, high fevers, and severe arthritis. These patients may experience spontaneous remissions during the few weeks that it takes to confirm the diagnosis. Therefore, these patients may never require any therapy. Predicting the natural history of the disease for the patient during the course of therapy is important. In the ideal setting, the treated patients should be compared to untreated patients, but most investigators are reluctant to include an untreated group.

The ethics of withholding corticosteroids from patients with severe disease is a common concern in treatment trials. Trials comparing corticosteroids to placebo do not include patients with severe disease. Those patients are considered ineligible for study because they may develop end organ failure, or even die [2]. These patients constitute a variable portion of the sarcoidosis population $[3,4]$. In a recent multicentre trial of corticosteroids for acute sarcoidosis, $20 \%$ of patients were considered to be ineligible for randomization due to worsening disease over a 6month observation period [5]. Thus, prednisone was only studied in less severely ill patients. Choosing patients who may never require treatment hinders researchers from determining drug efficacy.

The relatively small number of patients in any one centre makes it difficult to pick and choose patients. Most investigators tend to treat most available patients and then discuss a postintervention analysis. This approach has been reported by several groups, including HuNNINGHAKE et al. [3] from Iowa, USA, Gottlieb et al. [4] from Philadel-

Dept of Medicine, University of Cincinnati Medical Center, Cincinnati, $\mathrm{OH}$, USA.

Correspondence: R.P. Baughman, University of Cincinnati Medical Center, 231 Bethesda Avenue, Room 6004, Cincinnati, OH 45267-0564, USA. Fax: 5135580360. phia, USA, and Rizzato et al. [6] from Italy. All three centres reported the numbers of patients treated, the number who could be withdrawn from therapy, and the number that relapsed. The number treated initially varied from $34-65 \%$, the number who could be withdrawn from steroids ranged $47-82 \%$, and the number who relapsed when steroids were stopped ranged $14-83 \%$. This wide variation highlights the difficulty of studying all patients with sarcoidosis.

Corticosteroids have such an obvious effect on the patient that a placebo control trial would be difficult to conceive. Instead of randomizing between corticosteroid and placebo, the strategy of demonstrating steroid sparing has been studied. In this situation, patients who are stable but requiring high doses of corticosteroids ( $>10 \mathrm{mg} \cdot \mathrm{day}^{-1}$ prednisone or prednisolone) are evaluated. In this setting, patients receive the new agent and are observed over the next 6-12 months. Some patients will improve over the next year, however, others will remain stable but will have their dose of corticosteroids reduced or discontinued. In open label studies, the improvement over the next year is attributed to the drug [7]. However, the patient may have already experienced remission but the physician was slow to taper the corticosteroids. For the patient who remains stable during the withdrawal, it may not be that the agent is steroid sparing. Perhaps the patient did not need any further drug.

One research interest has been in patients with chronic disease. Patients with active disease for $>2$ yrs should provide a more homogenous group. Defining chronic disease remains problematic. The presence of an abnormality such as fibrosis does not mean that the patient still has active inflammation. This fibrosis may be seen in the chest roentgenogram or in the eyes (dry eyes or glaucoma), or scars on the skin. To determine whether the inflammation has resolved, markers for disease activity have been proposed. None of these is perfect. An increased angiotensin converting enzyme (ACE) level or increased percentage of lymphocytes in the bronchoalveolar lavage (BAL) fluid $\geq 2$ yrs after initial presentation implies continued activity, but many patients with progressive disease have a normal ACE level. The ratio of CD4:CD8 lymphocytes decreases from $>3.5$ towards the normal range of $1-2.2$ as sarcoidosis spontaneously resolves [8]. However, a low CD4:CD8 ratio has been reported in some patients with chronic disease [9]. The need for continued therapy can be considered as a measure of persistent activity. This approach has some limitations. Some patients with advancing disease will refuse further therapy. Other patients may find corticosteroids helpful even when there is no further progression of the disease. Some physicians advocate 
long-term, low-dose corticosteroids even when there is no evidence of continued activity [10].

A recent paper by LEwIs et al. [11] analysed 10 patients with chronic sarcoidosis treated with azathioprine. The authors found that only 2 patients had significant, sustained response to the drug. They concluded that the role for azathioprine was limited. However, 5 of the patients remained stable and several of the patients were able to reduce their dose of corticosteroids.

A more optimistic note for azathioprine is reported by MÜLLER-QUeRNHEIM et al. [12] in this issue of the European Respiratory Journal. The author found that all 11 patients with chronic sarcoidosis treated with azathioprine and low-dose prednisolone showed some improvement. This study is different for several reasons. The authors chose to give all patients azathioprine plus corticosteroids. Thus, it is difficult to be sure how much benefit should be attributed to corticosteroids versus azathioprine. The high response rate may be due to the drug combination; however, there is no definite proof that these patients would not have responded to a prolonged trial of corticosteroids alone. What is interesting is that all of the patients improved. This is a higher success rate than the current authors have encountered in treating patients with chronic disease $[7,13]$. This would suggest that MüLLER-QUERNHEIM et al. [12] were able to identify patients with active disease. Their BAL findings in selected patients support the idea that these patients initially had active inflammation and that the inflammation improved with therapy.

The chronic patient group may be better to study for several reasons. Firstly, their incidence of spontaneous remission is much lower and the clinical course is more predictable. The sudden appearance of another organ manifestation is less likely. These patients are more likely to be compliant, since they understand the value of seeking alternative agents. One of the current authors' difficulties in studying acute disease concerns the significant number of patients who sustained a good short-term response to corticosteroids but were subsequently lost to short-term follow-up. They may return several months later, either worse because the drug was discontinued or experiencing side effects from the corticosteroids. In contrast, many chronic patients possess a high motivation to discontinue corticosteroids, and these patients are more agreeable to continued use of a slow acting agent. The need for drugs other than corticosteroids seems more important in the chronic patient since the morbidity of corticosteroids is more prominent for these patients.

As open label trials like that of MüLLER-QUERNHEIM et al. [12] and LeWIS et al. [11] demonstrate, there is a range in the response to an individual agent. Therefore, any clinical trial needs to include a standard approach for assessing disease activity and the indication for corticosteroids. If one physician (or group) is treating all patients similarly in a randomized, double blind trial, then the effect of physician bias may be negated by the placebo group. However, each centre tends to have its own approach to corticosteroid use. In a multicentre trial, a standard approach to corticosteroid changes must be used.

Proving that patients still have active disease is also crucial for any therapeutic trial. As in cancer trials, the clinical investigator must be examining "measurable" disease. One example is the vital capacity. Patients with sarcoidosis who experience significant decreases in vital capacity have proven that their disease is still active, not just in a fibrotic, but stable stage. Other examples of measurable activity include skin lesions such as lupus pernio or the white blood cells seen in the anterior chamber of a patient with uveitis. These patients with active progressing disease are more likely to demonstrate a response to therapy.

As the list of potential agents for sarcoidosis increases, so should the clinical trials. The investigator has to choose the patients carefully. A good trial should include a homogenous population using a consistent protocol for corticosteroids, and the patients should have active disease. Such a study is likely to prove (or disprove) the effectiveness of the agent for sarcoidosis. These studies will probably be multicentre and are expensive. However, the information gathered from the current open label trials supports the concept of new multicentre studies designed to evaluate new treatment modalities for sarcoidosis.

\section{References}

1. Baughman RP, Lower EE. Steroid-sparing alternative treatments for sarcoidosis. Clin Chest Med 1997; 18: 853-864.

2. Baughman RP, Winget DB, Bowen EH, Lower EE. Predicting respiratory failure in sarcoidosis patients. Sarcoidosis 1997; 14: 154-158.

3. Hunninghake GW, Gilbert S, Pueringer R, et al. Outcome of the treatment for sarcoidosis. Am J Respir Crit Care Med 1994; 149: 893-898.

4. Gottlieb JE, Israel HL, Steiner RM, Triolo J, Patrick H. Outcome in sarcoidosis: the relationship of relapse to corticosteroid therapy. Chest 1997; 111: 623-631.

5. Gibson GJ, Prescott RJ, Muers MF, et al. British Thoracic Society Sarcoidosis study: effects of long term corticosteroid treatment. Thorax 1996; 51: 238-247.

6. Rizzato G, Montemurro L, Colombo P. The late follow-up of chronic sarcoid patients previously treated with corticosteroids. Sarcoidosis 1998; 15: 52-58.

7. Lower EE, Baughman RP. Prolonged use of methotrexate for sarcoidosis. Arch Intern Med 1995; 155: 846-851.

8. Ceuppens JL, Lacquet LM, Marien G, Demedts M, Van Den Eeckhout A, Stevens E. Alveolar T-cell subsets in pulmonary sarcoidosis: correlation with disease activity and effect of steroid treatment. Am Rev Respir Dis 1984; 129: 563-568.

9. Agostini C, Trentin L, Zambello R, et al. CD8 alveolitis in sarcoidosis: incidence, phenotypic characteristics, and clinical features. Am J Med 1993; 95: 466-472.

10. Johns CJ, Zachary JB, Ball WC. A ten year study of corticosteroid treatment of pulmonary sarcoidosis. Johns Hopkins Med 1974; 134: 271-283.

11. Lewis CJ, Ainslie GM, Bateman ED. Efficacy of azathioprine as second-line treatment in pulmonary sarcoidosis. Sarcoidosis Vasc Diffuse Lung Dis 1999; 16: 87-92.

12. Müller-Quernheim J, Kienast K, Held M, Pfeifer S, Costabel U. Treatment of chronic sarcoidosis with an azathioprine/prednisolone regimen. Eur Respir J 1999; 14: 1117-1122.

13. Baughman RP, Lower EE. Alternatives to corticosteroids in the treatment of sarcoidosis. Sarcoidosis 1997; 14: 121-130. 